\title{
A Novel Molecular Target in EGFR-Mutant Non- Small Cell Lung Cancer Treated With a Combination of Osimertinib and Pemetrexed
}

\section{Natsuki Takano}

Department of Pulmonary Medicine and Oncology, Graduate School of Medicine, Nippon Medical School

\section{Masahiro Seike ( $\nabla$ mseike@nms.ac.jp )}

Department of Pulmonary Medicine and Oncology, Graduate School of Medicine, Nippon Medical School

\section{Teppei Sugano}

Department of Pulmonary Medicine and Oncology, Graduate School of Medicine, Nippon Medical School

\section{Kuniko Matsuda}

Department of Pulmonary Medicine and Oncology, Graduate School of Medicine, Nippon Medical School

\section{Kakeru Hisakane}

Department of Pulmonary Medicine and Oncology, Graduate School of Medicine, Nippon Medical School

\section{Akiko Yoshikawa}

Department of Pulmonary Medicine and Oncology, Graduate School of Medicine, Nippon Medical School

\section{Shinji Nakamichi}

Department of Pulmonary Medicine and Oncology, Graduate School of Medicine, Nippon Medical School

\section{Rintaro Noro}

Department of Pulmonary Medicine and Oncology, Graduate School of Medicine, Nippon Medical School

\section{Akihiko Gemma}

Department of Pulmonary Medicine and Oncology, Graduate School of Medicine, Nippon Medical School

\section{Research Article}


Keywords: Non-small-cell lung cancer, Epidermal growth factor receptor, Osimertinib, Pemetrexed, Pololike kinase 1

Posted Date: March 11th, 2021

DOI: https://doi.org/10.21203/rs.3.rs-257308/v1

License: (c) (1) This work is licensed under a Creative Commons Attribution 4.0 International License. Read Full License 


\section{Abstract}

Overcoming acquired resistance to the epidermal growth factor receptor tyrosine kinase inhibitor (EGFRTKI) osimertinib is currently an important clinical issue in treating EGFR-mutant non-small cell lung cancer (NSCLC) patients. EGFR-TKI may synergize with chemotherapy and offer novel treatment strategies for overcoming acquired resistance. Here, we evaluate the therapeutic potential of combining osimertinib with pemetrexed and clarify the underlying molecular mechanisms. Gene expression microarrays were used to assess gene expression on exposure to osimertinib in the presence or absence of pemetrexed and we established cell lines resistant to osimertinib as well as those resistant to osimertinib+pemetrexed in order to explore mechanisms of resistance. Osimertinib+pemetrexed treatment significantly delayed the emergence of resistance relative to monotherapy in vitro and in vivo. Microarray analysis revealed significantly downregulated expression of the anti-apoptotic gene PLK1 in cells treated with osimertinib+pemetrexed. Consistent with this, cell lines resistant to osimertinib or to osimertinib+pemetrexed, exhibited overexpression of PLK1. Accordingly, PLK1 inhibition by siRNA or PLK1 inhibitor volasertib inhibited proliferation by inducing apoptosis in these resistant cell lines. Blocking PLK1 contributes to mediating the synergistic antiproliferative effect of osimertinib+pemetrexed in EGFR-mutant NSCLC cells. PLK1 overexpression may be a critical mechanism responsible for the acquired resistance of such mutants to osimertinib+pemetrexed.

\section{Introduction}

Recently developed anti-cancer treatments such as molecular-targeted drugs and immunotherapeutic agents have elicited dramatic responses in some advanced non-small cell lung cancer (NSCLC) patients 1. In particular, NSCLC patients with epidermal growth factor receptor (EGFR) activating mutations, such as the in-frame deletion [del(E746-A750)] in exon 19 or mutated exon 21 (L858R), show high sensitivity to EGFR-tyrosine kinase inhibitors (TKIs) ${ }^{2-6}$. The FLAURA phase III trial reported that progression-free survival (PFS) and overall survival (OS) were significantly longer in treatment-naive EGFR-mutant NSCLC patients treated with the 3rd generation EGFR-TKI osimertinib relative to those treated with first-

generation drugs of this type ${ }^{7}$. However, initially susceptible NSCLCs inevitably acquire resistance to osimertinib treatment and thus there is an urgent unmet need for novel treatment strategies to overcome or delay such acquired resistance to EGFR-TKIs.

Previous studies have documented synergistic effects when treating NSCLC with a combination of EGFRTKI and cytotoxic agents ${ }^{8-10}$. Phase III clinical trials indicated that combining gefitinib with concurrent chemotherapy resulted in improved PFS of patients with untreated advanced NSCLC harboring the appropriate EGFR mutations ${ }^{11,12}$. Currently, ongoing phase II and III trials are comparing osimertinib with osimertinib+chemotherapy such as cisplatin/carboplatin and pemetrexed ${ }^{13}$. Nonetheless, the molecular mechanisms responsible for synergistic effects of combination treatments in NSCLC are not completely understood, but could result in improved therapeutic approaches. We have previously contributed to pivotal phase II-III clinical trials conducted by the North East Japan Study Group in EGFR-mutant NSCLC 
patients $2,11,13$. In addition, our previous studies have identified promising therapeutic targets for overcoming resistance to targeted inhibitors in NSCLC patients with driver oncogenes ${ }^{14-17}$.

In the present study, we employed two different NSCLC cell lines harboring EGFR mutations to investigate possible mechanisms responsible for the superior efficacy of combination therapy with osimertinib+pemetrexed. Here, we undertook gene expression profiling to identify molecular mechanisms contributing to the success of combination therapy. To this end, we established NSCLC cell lines resistant to osimertinib alone or to the combination of osimertinib and pemetrexed as models to investigate potential therapeutic strategies for overcoming resistance to combination therapy.

\section{Materials And Methods}

\section{Cell culture}

Two human lung adenocarcinoma cell lines were employed in the present study. PC-9 has an EGFR exon 19 deletion. It was provided by Immuno-Biological Laboratories (Gunma, Japan). NCl-H1975 (H1975) with an exon 21 L858R mutation and secondary exon 20 T790M mutation was purchased from the American Type Culture Collection (Manassas, VA). These cell lines were cultured in RPMI-1640 (FUJIFILM, Osaka, Japan) containing 10\% fetal bovine serum (FBS; Biowest, Nuaille, France) and 1\% penicillin and streptomycin (FUJIFILM) at $37^{\circ} \mathrm{C}$ in a $5 \% \mathrm{CO}_{2}$ incubator. The cell lines were obtained in 2011 and 2014, expanded and cryopreserved, and one aliquot of each was thawed for this study. No cell line authentication was performed by the authors. All cells were routinely screened for the absence of mycoplasma.

\section{Drugs and cell viability assays}

Osimertinib, pemetrexed, and the Polo-like kinase 1 (PLK1) inhibitor volasertib were purchased from Selleck Chemicals (Houston, TX). To assess their sensitivity to drugs in vitro, cells were plated in 96-well tissue culture plates at 5,000 cells/well and incubated at $37^{\circ} \mathrm{C}$ for 24 hours. Cells were then incubated with titrated concentrations of drugs $(0.001,0.01,0.1,1,10 \mu \mathrm{M})$ or vehicle (DMSO) at $37^{\circ} \mathrm{C}$ for 72 hours. We estimated cell numbers using the Counting Kit-8 (Dojindo, Kumamoto, Japan). The half maximal inhibitory concentration $\left(\mathrm{IC}_{50}\right)$ value for the drugs tested was defined as the concentration of osimertinib or pemetrexed or combinations thereof required for a $50 \%$ reduction of cell growth. Each experiment was individually performed three times. We calculated the corrected absorbance of each sample and compared it with that of the control group, in accordance with the protocol provided by the manufacturer of the kits.

\section{Western blotting}

Protein extraction and Western blotting were performed as previously described ${ }^{14,15}$. The membranes were incubated with antibodies against PLK1, TS, EGFR, phosphorylated EGFR ( $p$-EGFR), AKT, p-AKT, ERK, 
p-ERK, BIM and cleaved PARP, all of which were purchased from Cell Signaling Technology (Danvers, MA). An antibody against GAPDH were obtained from Santa Cruz Biotechnology (Santa Cruz, CA).

\section{Apoptosis assays}

Apoptosis assays were performed using the Annexin \-FITC Apoptosis Detection Kit (Nacalai Tesque, Inc, Kyoto, Japan) and measured by flow cytometry. Cells were harvested by treating with trypsin-EDTA, washed with PBS, and centrifuged at $1500 \mathrm{rpm}$ for 3 minutes. The cell pellets $\left(5.0 \times 10^{5}\right.$ cells) were resuspended in $100 \mu \mathrm{L}$ of binding buffer containing $5 \mu \mathrm{L}$ of Annexin $\otimes-f l u o r e s c e i n$ isothiocyanate and 5 $\mu \mathrm{L}$ of propidium iodide, incubated for 15-30 minutes in the dark on ice, after which fluorescence was quantified on a BD FACSVerse flow cytometer. Data were processed with FACSuite software (Becton Dickinson, Franklin Lakes, $\mathrm{NJ}$ ).

\section{RNA extraction and quantitative real-time reverse transcription-PCR}

Total RNA was extracted from cultured cells using TRIzol Reagent (Thermo Fisher Scientific, Waltham, MA) as previously described ${ }^{39}$. The RNA was reverse-transcribed to cDNA using a ReverTra Ace qPCR RT Master Mix (Toyobo, Osaka, Japan) according to the manufacturer's instructions. The primers, cDNA, and THUNDERBIRD Probe qPCR Mix (Toyobo) were mixed together, and qPCR was performed using the 7500 Fast Real-Time PCR System (Applied Biosystems, San Francisco, CA). GAPDH expression was taken as the standard against which to normalize relative expression of the genes of interest. Plk1 and TS expression was quantified using the TaqMan Gene Expression Assay (Thermo Fisher Scientific) and presented as $2^{-\triangle \Delta} C_{\mathrm{t}}$ values ${ }^{39}$.

\section{Microarray analysis}

Gene expression microarray analysis was performed using the GeneChip Human Gene 2.0 Sense Target array (Affymetrix, Santa Clara, CA) according to the manufacturer's protocol. Microarray data have been deposited in NCBI's Gene Expression Omnibus (GEO; http://www.ncbi.nlm.nih.gov/geo/) and are accessible through a GEO series accession number GSE165029.

\section{Transfection of small-interfering RNA (siRNA)}

SiRNA experiments were performed using Silencer Select Negative Control siRNA as the negative control. Pre-Designed Silencer Select siRNAs were used to knock down PLK1 and TS (Thermo Fisher Scientific). SiRNA and Lipofectamine RiMAX Reagent were dissolved in Opti-MEM Media (Thermo Fisher Scientific) at a final concentration of siRNA complexes of $20 \mathrm{nM}$. Next, we replaced the transfection medium after 6 hours, and finally incubated cells at $37^{\circ} \mathrm{C}$ for a further 48 hours.

\section{Tumor cell implantation experiments}

Female severe combined immunodeficient (SCID)-Beige mice (CAnN.Cg-Foxn $1^{\text {nu }} / \mathrm{CrlCrlj}$ ) were purchased at 5 weeks of age from Charles River Laboratories Japan Inc., Kanagawa, Japan. A total of $5.0 \times 10^{6}$ PC-9 
cells was suspended in $200 \mu \mathrm{l}$ of Matrigel (CORNING Inc., Corning, NY) / PBS (Nacalai Tesque, Inc) and was injected into the subcutaneous area of 6-week-old mice. When tumor volumes reached an average size of $200 \mathrm{~mm}^{3}$, the mice were divided into four groups ( $\mathrm{n}=6$ for each group): vehicle control (per os (p.o.), once a day), pemetrexed (intraperitoneal (i.p.), at $100 \mathrm{mg} / \mathrm{kg}$, twice a week), osimertinib (p.o., at 1.0 $\mathrm{mg} / \mathrm{kg}$, once a day), or both osimertinib (p.o., $1.0 \mathrm{mg} / \mathrm{kg}$, once a day) and pemetrexed (intraperitoneal (i.p.), at $100 \mathrm{mg} / \mathrm{kg}$, twice a week). Treatments were administered until the end of the 29-day observation period. Body weights were measured every week. We estimated tumor volumes $(V)$ twice a week by caliper measurements of the width $(W)$ and length $(L)$ of each tumor $\left(W^{2} \times L / 2\right)$. Extraction of tumor proteins for immunohistochemical analysis was performed using samples from mice at the end of the 29-day treatment period. The study protocol was approved by the Ethics Committee on the Use of Laboratory Animals, KAC Co., Ltd (Shiga, Japan) (approval no. 20-0313). All experiments on live vertebrates were carried out in accordance with relevant guidelines and regulations. The study was performed in compliance with the Animal research: Reporting of in vivo experiments (ARRIVE) guidelines.

\section{Immunohistochemical analysis}

PLK1 expression was detected in deparaffinized formalin-fixed, paraffin-embedded tissue sections ( $3 \mu \mathrm{m})$ using a PLK/PLK1 antibody. Immunohistochemical analysis (IHC) was performed as previously described ${ }^{40}$. The sections were stained overnight at $4^{\circ} \mathrm{C}$ with rabbit anti-human PLK/PLK1 antibody (ReyBiotech, Norcross, GA) at a final dilution of 1:500. For human specimens, written informed consent was obtained from the patient, and the specimens were collected in accordance with the Declaration of Helsinki 2013. The study protocol was approved by the Ethics Committee Review Board at Nippon Medical School Hospital (approval no. 25-05-299 and B-2020-286).

\section{Statistical analysis}

Data were expressed as the mean \pm standard error (SE) of three independent experiments and evaluated with Student's $t$ test. A $P$ value of $<0.05$ was considered to be significant.

\section{Results}

\section{Increased apoptotic activity caused by the combination of osimertinib and pemetrexed in NSCLC cells with EGFR mutations}

We first examined the effects of osimertinib alone or pemetrexed alone on the proliferation of PC-9 and H1975 cells, which both harbor EGFR mutations. These cell lines were highly sensitive to osimertinib, with $\mathrm{IC}_{50}$ values of $0.069 \pm 0.0079$ and $0.085 \pm 0.016 \mu \mathrm{mol} / \mathrm{L}$, respectively. Pemetrexed was also a potent inhibitor of proliferation with $\mathrm{IC}_{50 \mathrm{~s}}$ of $0.080 \pm 0.0053 \mu \mathrm{mol} / \mathrm{L}$ and $0.080 \pm 0.013 \mu \mathrm{mol} / \mathrm{L}$, respectively.

Next, we compared anti-proliferative effects of combined treatment with osimertinib and pemetrexed, using the $\mathrm{IC}_{50}$ doses established above. We found that the anti-proliferative effect of combined treatment was significantly greater than with either alone for both PC-9 and H1975 cells (Fig. 1A). We examined the 
effects of drug combinations on apoptosis and found increased levels of cleaved PARP and BIM proteins in cells treated with osimertinib+pemetrexed (Fig. 1B). Osimertinib+pemetrexed caused increased

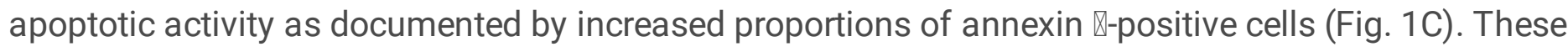
results indicate that combining osimertinib with pemetrexed increases apoptosis and thus leads to enhanced anti-proliferative activity of NSCLC cells with EGFR mutations.

\section{Long-lasting anti-tumor effect of osimertinib combined with pemetrexed in NSCLC cells with mutated EGFR}

We assessed whether a combination of osimertinib with pemetrexed prevents the acquisition of resistance to the former. PC-9 and H1975 cells were exposed to osimertinib with or without pemetrexed at half the $\mathrm{IC}_{50}$ dose of the drugs, and then their concentrations were slowly increased with time in culture. After 2 months, the $\mathrm{IC}_{50}$-values for osimertinib alone and the combination of osimertinib and pemetrexed in PC-9 cells were $3.34 \pm 0.29 \mu \mathrm{mol} / \mathrm{L}$ and $<0.001 \mu \mathrm{mol} / \mathrm{L}$, respectively (Fig. $2 \mathrm{~A}$ ). In H1975, these $\mathrm{IC}_{50^{-}}$ values were $3.58 \pm 0.82 \mu \mathrm{M}$ and $0.0072 \pm 0.0041 \mu \mathrm{M}$, respectively, after 3 months (Fig. 2B). These results showed that combining osimertinib with pemetrexed significantly hinders the emergence of osimertinib resistance relative to monotherapy in these EGFR-mutant NSCLC cells.

We further investigated whether osimertinib+pemetrexed combination therapy enhanced cytotoxicity to PC-9 in vivo, in mouse xenograft models. The animals exhibited none of the toxicity otherwise seen with high doses and regularly gained body weight (data not shown). Osimertinib combined with pemetrexed (group 3) inhibited the growth of tumors to a significantly greater degree than pemetrexed alone (group 1) or osimertinib alone (group 2) over this time course (Fig. 2C). Thus, combining osimertinib with pemetrexed mediated enhanced in vivo antitumor effects against xenografts harboring the EGFR mutation, consistent with the results obtained in vitro.

\section{Role of thymidylate synthase for the apoptotic activity resulting from combination therapy with osimertinib and pemetrexed}

To investigate the molecular mechanisms of the synergistic effects of combined osimertinib and pemetrexed, we assessed the expression of the transcription factor thymidylate synthase (TS) and EGFR signal pathway mediators at the protein level in PC-9 and H1975 cells. The levels of phosphorylated EGFR, AKT, and ERK were markedly reduced by osimertinib, as determined by Western blotting (Fig. 3A). A previous study reported that EGFR-TKI irreversibly inhibit TS expression, which is well known as a therapeutic target of pemetrexed ${ }^{18}$. Accordingly, we evaluated the effect of osimertinib on TS expression and found that it was significantly reduced at both the mRNA and protein level in PC-9 and H1975 cells in a dose-dependent manner (Fig. 3B, C). Thus, osimertinib-inhibited TS expression resulted in a synergistic antiproliferative interaction with pemetrexed in these EGFR-mutant NSCLC cells. We next examined whether the inhibition of TS protein expression enhanced the pro-apoptotic activity of pemetrexed in these cells. However, we found that TS-specific siRNA treatment did not enhance the apoptotic activity in PC-9 cells treated with pemetrexed, as assessed by the Annexin $V$ flow cytometry assay (Fig. 3D). 


\section{The combination of osimertinib and pemetrexed enhances apoptosis via a PLK1 signaling pathway}

To clarify the molecular mechanisms underlying the enhanced apoptotic activity of the combination therapy, we used GeneChip microarrays to profile gene expression in PC-9 and H1975 cells treated with osimertinib with or without pemetrexed (Fig. 4A). This revealed that Polo-like kinase 1 (PLK1), AURKA and $\mathrm{HIST} 1 \mathrm{H} 2$ were significantly downregulated in both cell lines treated with osimertinib and pemetrexed. Pathway analysis was performed to provide a perspective on the apoptotic function of these three genes. PLK1 as well as AURKA were shown to be important genes associated with apoptotic activity (Fig. 4B). Previous studies had indicated that PLK1 plays an important role in cell cycle control and apoptosis in human cancer cells ${ }^{19-21}$. We confirmed that treatment with the combination of osimertinib and pemetrexed resulted in significant downregulation of PLK1 in PC-9 and H1975, as documented both by qRT-PCR and Western blotting (Fig. 4C). Furthermore, we quantified PLK1 protein in PC-9 and H1975 after the cell lines had been treated with osimertinib and pemetrexed for 1-3 months. Reduced PLK1 protein expression and increased cleaved PARP was still seen in PC-9 and $\mathrm{H} 1975$ cells treated with both drugs for 2-3 months. In contrast, the levels of these two proteins were already increased in cells treated with osimertinib alone (Fig. 4D). We next analyzed PLK1 expression by immunohistochemical staining in tumors derived from PC-9 in the mouse xenograft model. We found that the number of PLK1-negative tumor cells was significantly higher in the osimertinib and pemetrexed-treated group (Fig. 4E). To elucidate the potential mechanisms of PLK1 involvement in regulating apoptotic activity, we evaluated whether knocking down PLK1 would induce apoptosis of these EGFR-mutant NSCLC cells. Indeed, treatment with PIk1-specific siRNA did enhance apoptosis as assessed by flow cytometry and Western blotting (Fig. 4F, G).

These findings suggest that treatment with a combination of osimertinib+pemetrexed delayed the upregulation of PLK1 and resulted in enhanced induction of apoptosis, thereby delaying the emergence of drug-resistance relative to monotherapy.

\section{PLK1 overexpression in osimertinib+pemetrexed-resistant EGFR-mutant cells and patients}

We have established osimertinib-resistant PC-9 (PC-9 OsiR) and osimertinib+pemetrexed-resistant PC-9 (PC-90siPEMR) cell lines (Fig. 5A). In both of these, we found that p-EGFR was markedly reduced, but pAKT and p-ERK were expressed, suggesting the involvement of an EGFR-independent signaling pathway (Fig. 5B). In contrast, PLK1 was strongly overexpressed in both PC-90siR and PC-90siPEMR (Fig. 5C). Importantly, we also investigated PLK1 expression in EGFR-mutant NSCLC samples from a patient before and after osimertinib+pemetrexed therapy. This patient had previously received several different EGFRTKI and chemotherapies including gefitinib, erlotinib, afatinib, osimertinib, and pemetrexed 22 . Interestingly, increased PLK1 expression was observed after resistance to osimertinib and pemetrexed had been acquired (Fig. 5D). Thus, PLK1 upregulation is implicated in the acquired resistance to osimertinib+pemetrexed in EGFR mutants, both in cell lines and in a patient, independent of EGFR signaling. 


\section{PLK1 inhibitors induce apoptosis of osimertinib-resistant cell lines, thus overcoming resistance to this drug}

Finally, we tested whether PLK1 inhibition induced apoptosis of EGFR mutant NSCLC cells in order to evaluate the potential of PLK inhibitors as novel therapeutic agents. PLK1 knockdown by PIk1-specific siRNA resulted in increased numbers of Annexin V-positive cells and increased expression of apoptotic markers, suggesting enhanced apoptotic activity (Fig. 6A, B). We next examined whether PLK1 inhibitors could overcome osimertinib+pemetrexed resistance in EGFR-mutant NSCLC cells. We selected volasertib (B16727) as the PLK-1 inhibitor in this experiment. A previous study had reported that volasertib could overcome resistance to the EGFR-TKI erlotinib in NSCLC cells ${ }^{23}$. We found that volasertib significantly inhibited the proliferation of both PC-90siR and PC-90siPEMR cells (Fig. 6C). We also found increased levels of cleaved PARP and BIM proteins in cells treated with volasertib, reflecting the activation of apoptosis (Fig. 6D). PLK1 inhibitors may therefore represent promising agents for treating EGFR-mutant NSCLC resistant to osimertinib combined with pemetrexed.

\section{Discussion}

In this study, we demonstrated that the combination of osimertinib and pemetrexed induced more apoptosis and enhanced anti-tumor effects compared to monotherapy in EGFR-mutant NSCLC in vitro and in vivo. Several EGFR-dependent or independent osimertinib resistance mechanisms are known, including MET amplification, C797S mutation, HER2 amplification, PIK3CA and RAS mutations ${ }^{24}$. However, specific therapeutic strategies to overcome resistance to osimertinib in the clinical setting for curative EGFR-mutant NSCLC treatment have not yet been established. It has been proposed that combining EGFR-TKIs including gefitinib and osimertinib with cisplatin/carboplatin and pemetrexed in NSCLC patients might represent a strategy to overcome acquired resistance ${ }^{11-13}$. Our findings provide evidence for the feasibility of this approach in EGFR-mutant NSCLC patients.

We have ultimately identified PLK1 as a therapeutic target for combination therapy and as a promising treatment target for osimertinib-resistant tumors in EGFR-mutated NSCLC. PLK1 is highly overexpressed in various different malignancies including glioma, head and neck cancer, ovarian cancer, prostate cancer, breast cancer and NSCLC ${ }^{25-30}$. Increased PLK1 expression was correlated with poor prognosis in NSCLC and breast cancer ${ }^{31,32}$. PLK1 is one of a family consisting of 5 members, PLK1, $-2,-3,-4$ and -5 and has a key role in cell-cycle regulation, including mitotic entry, centrosome maturation, bipolar spindle maturation, activation of cyclin and cyclin-dependent kinases, spindle assembly and chromosome separation ${ }^{33,34}$. PLK1 also modulates DNA damage responses, including recovery from DNA damage checkpoints. PLK1 overexpression emphasizes its tumor-promoting activity. PLK1 inhibition has been shown to cause mitotic blockade and to result in apoptosis ${ }^{21,35,36}$. Therefore, PLK1 down-regulation induced by combination therapy of osimertinib+pemetrexed contributes to enhanced apoptosis and its inhibition might have therapeutic value for EGFR-mutant NSCLC patients. 
In this study, we showed that the PLK1 inhibitor volasertib has potential as a novel therapeutic agent for EGFR-mutant NSCLC patients. Volasertib was granted orphan drug designation for acute myeloid leukemia (AML) in 2014 after showing promising efficacy in clinical trials ${ }^{37,38}$. In lung cancer, erlotinibresistant T790M-negative EGFR-mutated NSCLC cells with epithelial-mesenchymal transition properties had higher sensitivity to volasertib, which caused G2/M arrest and apoptosis. Erlotinib-resistant T790Mpositive EGFR-mutated NSCLC cells had a higher sensitivity to the combination of erlotinib and volasertib than to either agent alone. PLK1 inhibition together with erlotinib treatment could enhance DNA damage and apoptosis because of inhibiting DNA repair via EGFR inhibition ${ }^{23}$. These results are consistent with our findings that EGFR-TKI-resistant cells are sensitive to volasertib independent of EGFR signaling. Our study demonstrates a possible pivotal role for volasertib in treating osimertinib-resistant or osimertinib+pemetrexed-resistant cells.

Our study had some limitations. We could evaluate PLK1 overexpression after osimertinib+pemetrexed therapy in only one clinical sample. In general, re-biopsy after EGFR-TKI therapy is difficult because of the small number of areas that can be biopsied. Further studies need to be performed to evaluate PLK1 expression after osimertinib+pemetrexed treatment using more clinical samples.

In conclusion, we found that PLK1 downregulation, associated with pro-apoptotic and anti-proliferative activity, is seminally involved in the synergistic effects of osimertinib+pemetrexed combination therapy for EGFR-mutated NSCLC both in vitro and in vivo. Furthermore, PLK1 overexpression may be a critical mechanism responsible for acquired resistance of EGFR-mutated NSCLC cells to osimertinib or both osimertinib and pemetrexed treatment. These results suggest that PLK1 inhibition might be a novel therapeutic strategy to overcome acquired resistance.

\section{Abbreviations}

NSCLC, non-small-cell lung carcinoma

EGFR, epidermal growth factor receptor

TKI, tyrosine kinase inhibitor

PFS, progression free survival

TS, thymidylate synthase

PLK1, Polo-like kinase 1

\section{Declarations}

\section{Acknowledgements}


We thank Mrs. M. Hirao of Nippon Medical School (Tokyo, Japan) for excellent technical assistance. We thank KAC Co., Ltd. for conducting animal experiments.

\section{Author information}

\section{Affiliations}

Department of Pulmonary Medicine and Oncology, Graduate School of Medicine, Nippon Medical School, Bunkyo-ku, Tokyo, Japan

Natsuki Takano, Masahiro Seike, Teppei Sugano, Kuniko Matsuda, Kakeru Hisakane, Akiko Yoshikawa, Shinji Nakamichi, Rintaro Noro \& Akihiko Gemma

\section{Contributions}

N.T and M.S wrote the main manuscript text and prepared Figures 1-6. All authors reviewed the manuscript.

\section{Corresponding Author}

Correspondence to Masahiro Seike.

\section{Ethics declarations}

\section{Competing interests}

This study was supported in part by a Clinical Rebiopsy Bank Project for Comprehensive Cancer Therapy Development from Ministry of Education, Culture, Sports, Science and Technology Supported Program for the Strategic Research Foundation at Private Universities (to A.G and M.S). M.S has received a commercial research grant from Eli Lilly Japan K.K and has received speakers' bureau honoraria from AstraZeneca and Eli Lilly Japan K.K.

\section{Additional information}

Publisher's note

Springer Nature remains neutral with regard to jurisdictional claims in published maps and institutional affiliations.

\section{References}

1. Takano, N. et al. Improvement in the survival of patients with stage IV non-small-cell lung cancer: Experience in a single institutional 1995-2017. Lung Cancer 131, 69-77, doi:10.1016/j.lungcan.2019.03.008 (2019). 
2. Maemondo, M. et al. Gefitinib or chemotherapy for non-small-cell lung cancer with mutated EGFR. N. Engl. J. Med. 362, 2380-2388, doi:10.1056/NEJMoa0909530 (2010).

3. Mitsudomi, T. et al. Gefitinib versus cisplatin plus docetaxel in patients with non-small-cell lung cancer harbouring mutations of the epidermal growth factor receptor (WJTOG3405): an open label, randomised phase 3 trial. Lancet Oncol. 11, 121-128, doi:10.1016/s1470-2045(09)70364-x (2010).

4. Zhou, C. et al. Erlotinib versus chemotherapy as first-line treatment for patients with advanced EGFR mutation-positive non-small-cell lung cancer (OPTIMAL, CTONG-0802): a multicentre, open-label, randomised, phase 3 study. Lancet Oncol. 12, 735-742, doi:10.1016/s1470-2045(11)70184-x (2011).

5. Park, K. et al. Afatinib versus gefitinib as first-line treatment of patients with EGFR mutation-positive non-small-cell lung cancer (LUX-Lung 7): a phase 2B, open-label, randomised controlled trial. Lancet Oncol. 17, 577-589, doi:10.1016/s1470-2045(16)30033-x (2016).

6. Wu, Y. L. et al. Dacomitinib versus gefitinib as first-line treatment for patients with EGFR-mutationpositive non-small-cell lung cancer (ARCHER 1050): a randomised, open-label, phase 3 trial. Lancet Oncol. 18, 1454-1466, doi:10.1016/s1470-2045(17)30608-3 (2017).

7. Soria, J. C. et al. Osimertinib in Untreated EGFR-Mutated Advanced Non-Small-Cell Lung Cancer. N. Engl. J. Med. 378, 113-125, doi:10.1056/NEJMoa1713137 (2018).

8. La Monica, S. et al. Combination of Gefitinib and Pemetrexed Prevents the Acquisition of TKI Resistance in NSCLC Cell Lines Carrying EGFR-Activating Mutation. J. Thorac. Oncol. 11, 1051-1063, doi:10.1016/j.jtho.2016.03.006 (2016).

9. Yoshimura, N. et al. Phase II study of a combination regimen of gefitinib and pemetrexed as first-line treatment in patients with advanced non-small cell lung cancer harboring a sensitive EGFR mutation. Lung Cancer 90, 65-70, doi:10.1016/j.lungcan.2015.06.002 (2015).

10. La Monica, S. et al. Third generation EGFR inhibitor osimertinib combined with pemetrexed or cisplatin exerts long-lasting anti-tumor effect in EGFR-mutated pre-clinical models of NSCLC. J. Exp. Clin. Cancer Res. 38, 222, doi:10.1186/s13046-019-1240-x (2019).

11. Hosomi, Y. et al. Gefitinib Alone Versus Gefitinib Plus Chemotherapy for Non-Small-Cell Lung Cancer With Mutated Epidermal Growth Factor Receptor: NEJ009 Study. J. Clin. Oncol. 38, 115-123, doi:10.1200/jco.19.01488 (2020).

12. Noronha, V. et al. Gefitinib Versus Gefitinib Plus Pemetrexed and Carboplatin Chemotherapy in EGFRMutated Lung Cancer. J. Clin. Oncol. 38, 124-136, doi:10.1200/jco.19.01154 (2020).

13. Asahina, H. et al. A Phase II Study of Osimertinib Combined With Platinum Plus Pemetrexed in Patients With EGFR-Mutated Advanced Non-Small-cell Lung Cancer: The OPAL Study (NEJ032C/LOGIK1801). Clin. Lung Cancer, doi:10.1016/j.cllc.2020.09.023 (2020).

14. Sugano, T. et al. Inhibition of ABCB1 Overcomes Cancer Stem Cell-like Properties and Acquired Resistance to MET Inhibitors in Non-Small Cell Lung Cancer. Mol. Cancer Ther. 14, 2433-2440, doi:10.1158/1535-7163.Mct-15-0050 (2015).

15. Kitamura, K. et al. MiR-134/487b/655 cluster regulates TGF- $\beta$-induced epithelial-mesenchymal transition and drug resistance to gefitinib by targeting MAGI2 in lung adenocarcinoma cells. Mol. 
Cancer Ther. 13, 444-453, doi:10.1158/1535-7163.Mct-13-0448 (2014).

16. Nakamichi, S. et al. Overcoming drug-tolerant cancer cell subpopulations showing AXL activation and epithelial-mesenchymal transition is critical in conquering ALK-positive lung cancer. Oncotarget 9, 27242-27255, doi:10.18632/oncotarget.25531 (2018).

17. Takahashi, A. et al. Ankyrin Repeat Domain 1 Overexpression is Associated with Common Resistance to Afatinib and Osimertinib in EGFR-mutant Lung Cancer. Sci. Rep. 8, 14896, doi:10.1038/s41598018-33190-8 (2018).

18. Takezawa, K. et al. Enhanced anticancer effect of the combination of BIBW2992 and thymidylate synthase-targeted agents in non-small cell lung cancer with the T790M mutation of epidermal growth factor receptor. Mol. Cancer Ther. 9, 1647-1656, doi:10.1158/1535-7163.Mct-09-1009 (2010).

19. Strebhardt, K. Multifaceted polo-like kinases: drug targets and antitargets for cancer therapy. Nat Rev Drug Discov 9, 643-660, doi:10.1038/nrd3184 (2010).

20. Craig, S. N., Wyatt, M. D. \& Mclnnes, C. Current assessment of polo-like kinases as anti-tumor drug targets. Expert Opin Drug Discov 9, 773-789, doi:10.1517/17460441.2014.918100 (2014).

21. Spänkuch-Schmitt, B., Bereiter-Hahn, J., Kaufmann, M. \& Strebhardt, K. Effect of RNA silencing of polo-like kinase-1 (PLK1) on apoptosis and spindle formation in human cancer cells. J. Natl. Cancer Inst. 94, 1863-1877, doi:10.1093/jnci/94.24.1863 (2002).

22. Tozuka, T. et al. Pembrolizumab and salvage chemotherapy in EGFR T790M-positive non-small-cell lung cancer with high PD-L1 expression. Onco Targets Ther. 11, 5601-5605, doi:10.2147/ott.S168598 (2018).

23. Wang, Y. et al. Polo-like kinase 1 inhibition diminishes acquired resistance to epidermal growth factor receptor inhibition in non-small cell lung cancer with T790M mutations. Oncotarget 7, 47998-48010, doi:10.18632/oncotarget.10332 (2016).

24. Leonetti, A. et al. Resistance mechanisms to osimertinib in EGFR-mutated non-small cell lung cancer. Br. J. Cancer 121, 725-737, doi:10.1038/s41416-019-0573-8 (2019).

25. Dietzmann, K., Kirches, E., von, B., Jachau, K. \& Mawrin, C. Increased human polo-like kinase-1 expression in gliomas. J. Neurooncol. 53, 1-11, doi:10.1023/a:1011808200978 (2001).

26. Knecht, R. et al. Prognostic significance of polo-like kinase (PLK) expression in squamous cell carcinomas of the head and neck. Cancer Res. 59, 2794-2797 (1999).

27. Weichert, W. et al. Polo-like kinase isoform expression is a prognostic factor in ovarian carcinoma. $B r$. J. Cancer 90, 815-821, doi:10.1038/sj.bjc.6601610 (2004).

28. Weichert, W. et al. Polo-like kinase 1 is overexpressed in prostate cancer and linked to higher tumor grades. Prostate 60, 240-245, doi:10.1002/pros.20050 (2004).

29. Donizy, P. et al. Augmented expression of Polo-like kinase 1 is a strong predictor of shorter cancerspecific overall survival in early stage breast cancer at 15-year follow-up. Oncol. Lett. 12, 1667-1674, doi:10.3892/ol.2016.4890 (2016). 
30. Wolf, G. et al. Prognostic significance of polo-like kinase (PLK) expression in non-small cell lung cancer. Oncogene 14, 543-549, doi:10.1038/sj.onc.1200862 (1997).

31. Medema, R. H., Lin, C. C. \& Yang, J. C. Polo-like kinase 1 inhibitors and their potential role in anticancer therapy, with a focus on NSCLC. Clin. Cancer Res. 17, 6459-6466, doi:10.1158/10780432.Ccr-11-0541 (2011).

32. King, S. I. et al. Immunohistochemical detection of Polo-like kinase-1 (PLK1) in primary breast cancer is associated with TP53 mutation and poor clinical outcom. Breast Cancer Res. 14, R40, doi:10.1186/bcr3136 (2012).

33. Glover, D. M., Hagan, I. M. \& Tavares, A. A. Polo-like kinases: a team that plays throughout mitosis. Genes Dev. 12, 3777-3787, doi:10.1101/gad.12.24.3777 (1998).

34. Nigg, E. A. Polo-like kinases: positive regulators of cell division from start to finish. Curr. Opin. Cell Biol. 10, 776-783, doi:10.1016/s0955-0674(98)80121-x (1998).

35. McCarroll, J. A. et al. Therapeutic targeting of polo-like kinase 1 using RNA-interfering nanoparticles (iNOPs) for the treatment of non-small cell lung cancer. Oncotarget 6, 12020-12034, doi:10.18632/oncotarget.2664 (2015).

36. Liu, X., Lei, M. \& Erikson, R. L. Normal cells, but not cancer cells, survive severe Plk1 depletion. Mol. Cell. Biol. 26, 2093-2108, doi:10.1128/mcb.26.6.2093-2108.2006 (2006).

37. Döhner, H. et al. Randomized, phase 2 trial of low-dose cytarabine with or without volasertib in AML patients not suitable for induction therapy. Blood 124, 1426-1433, doi:10.1182/blood-2014-03560557 (2014).

38. Kobayashi, Y. et al. Phase I trial of volasertib, a Polo-like kinase inhibitor, in Japanese patients with acute myeloid leukemia. Cancer Sci. 106, 1590-1595, doi:10.1111/cas.12814 (2015).

39. Seike, M. et al. Use of a cytokine gene expression signature in lung adenocarcinoma and the surrounding tissue as a prognostic classifier. J. Natl. Cancer Inst. 99, 1257-1269, doi:10.1093/jnci/djm083 (2007).

40. Seike, M. et al. AXL and GAS6 co-expression in lung adenocarcinoma as a prognostic classifier. Oncol. Rep. 37, 3261-3269, doi:10.3892/or.2017.5594 (2017).

\section{Figures}


$\mathbf{A}$
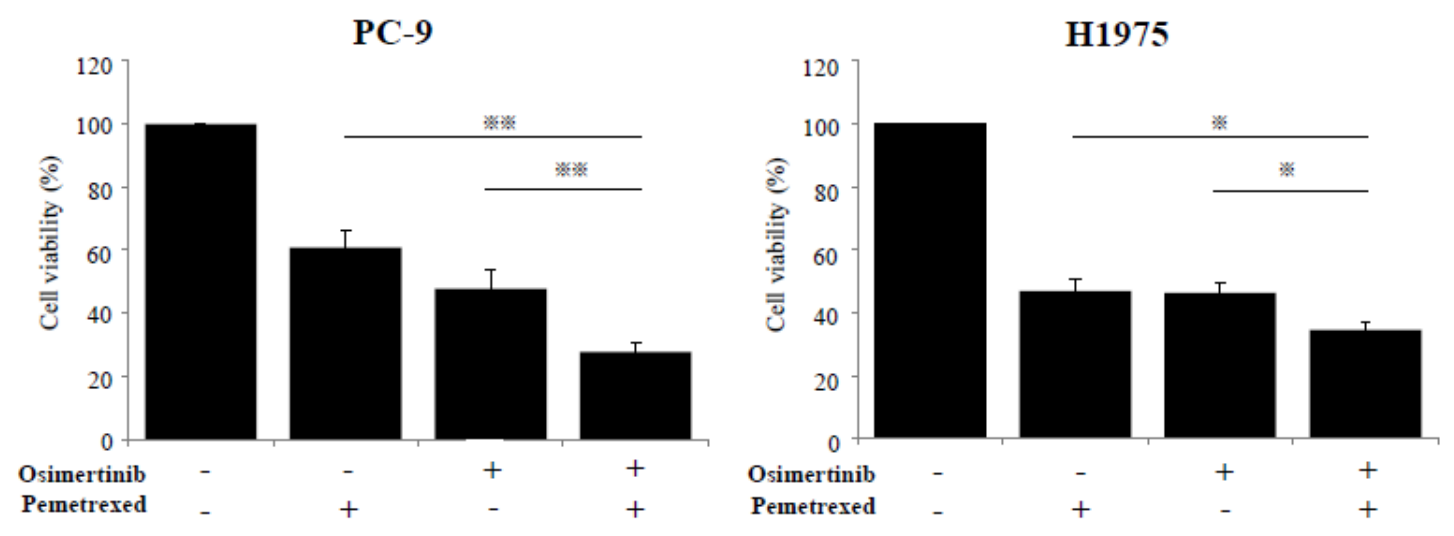

B

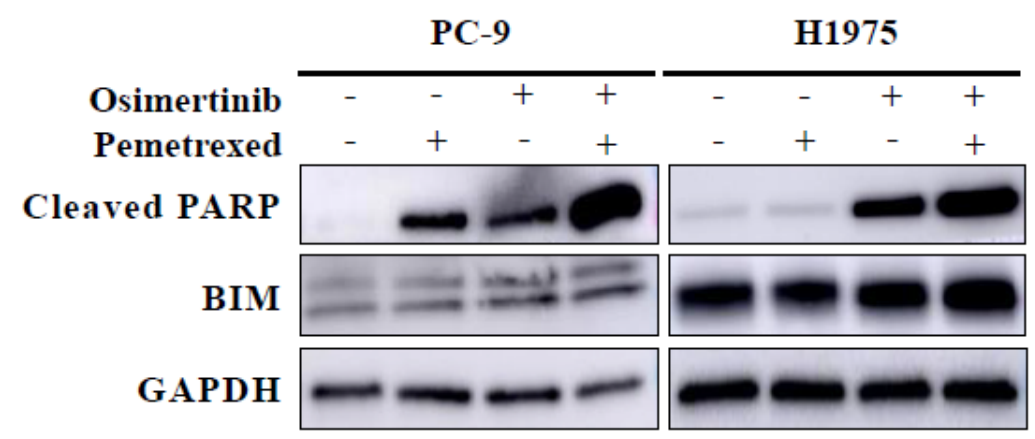

C
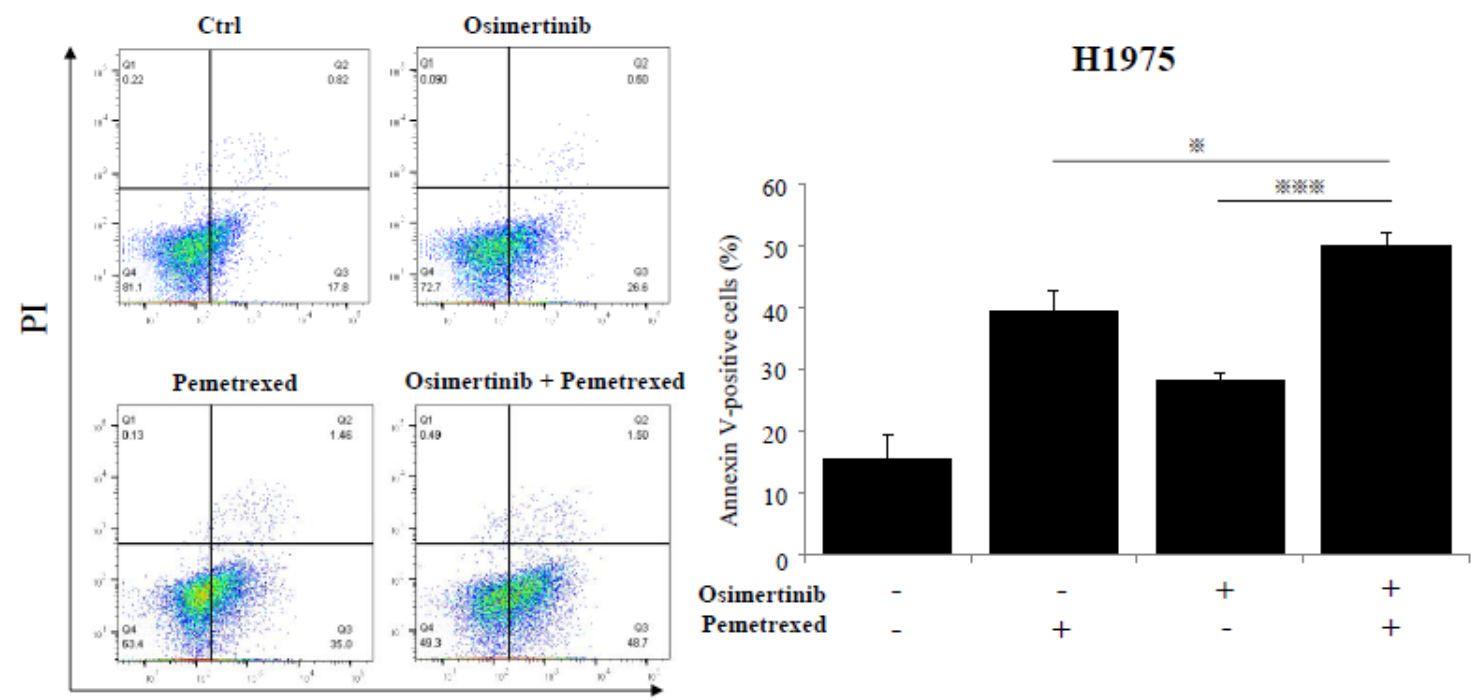

Annexin V

\section{Figure 1}

The effect of a combination of osimertinib and pemetrexed on inducing apoptosis of PC-9 and H1975 cells. A, PC- 9 and $\mathrm{H} 1975$ cells were incubated for 72 hours with osimertinib or pemetrexed or a combination of both at doses equivalent to their IC50 values. The antiproliferative effect of combination treatment was significantly increased relative to osimertinib or pemetrexed alone. Data are means \pm SEM from three independent experiments $(※ p<0.05, ※ * p<0.01)$. B, Western blots of proteins representing 
apoptosis markers. Higher expression of cleaved PARP and BIM is seen in PC-9 and H1975 cells treated with the drug combination. C, Apoptosis assay performed using an Annexin $\mathbb{D}$-FITC Apoptosis Detection Kit. Percentages of apoptotic cells are higher with combination treatment $(※ p<0.05, ※ ※ p<0.01, ※ ※ ※$ $p<0.001)$.
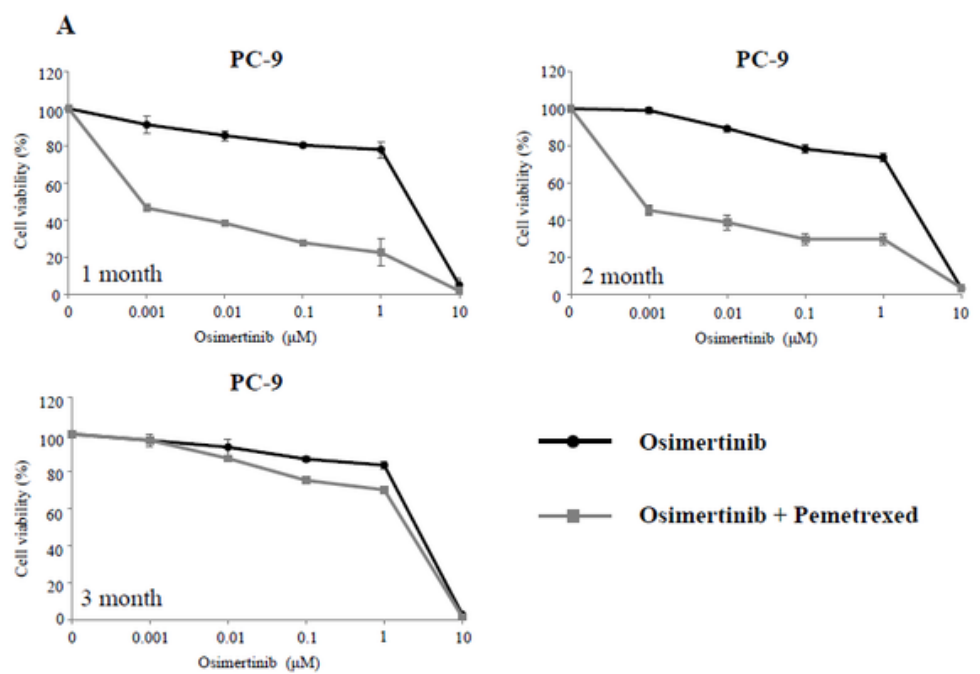

$\longrightarrow$ Osimertinib

$\longrightarrow$ Osimertinib + Pemetrexed

B
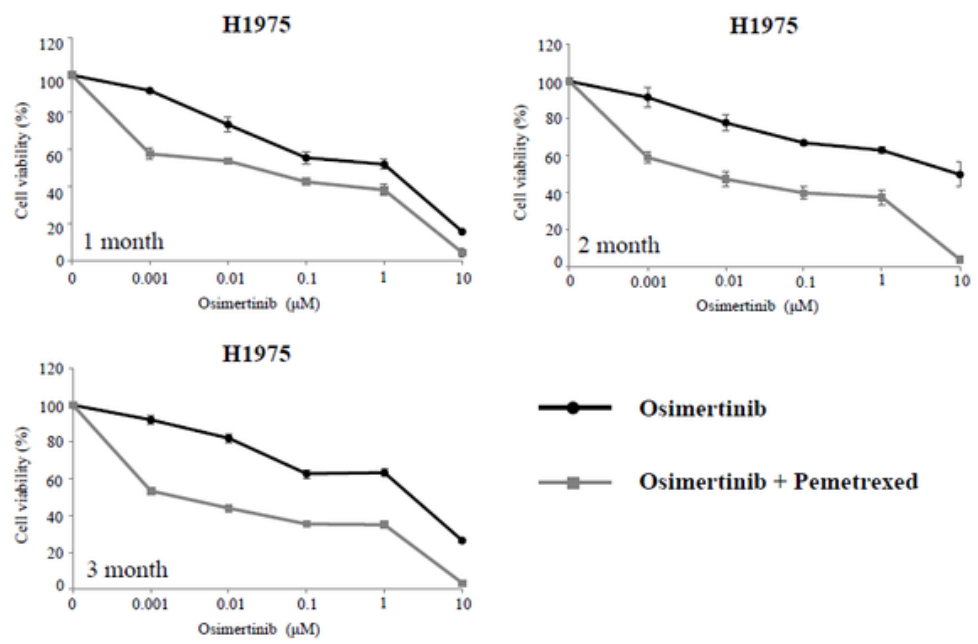

$\longrightarrow$ Osimertinib

C
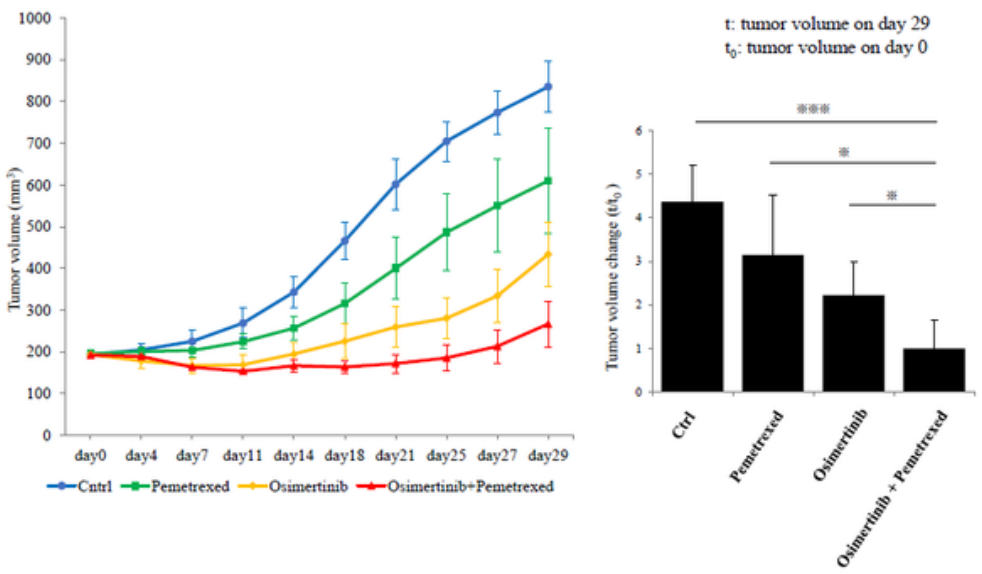

Figure 2 
Osimertinib in combination with pemetrexed delays the appearance of drug resistance with long-lasting effects. A, B, PC-9 and H1975 cells were exposed to half IC50- doses of drugs, either osimertinib alone or both osimertinib and pemetrexed, and then treated with increasing concentrations of osimertinib. Time to resistance was significantly delayed by combination treatment. The mean of 3 biologically independent samples per time point is shown. C, PC-9 cells were subcutaneously injected into mice and when the tumors had reached an average size of $200 \mathrm{~mm} 3$ the animals were treated with vehicle alone, osimertinib alone, pemetrexed alone or both osimertinib and pemetrexed.

A

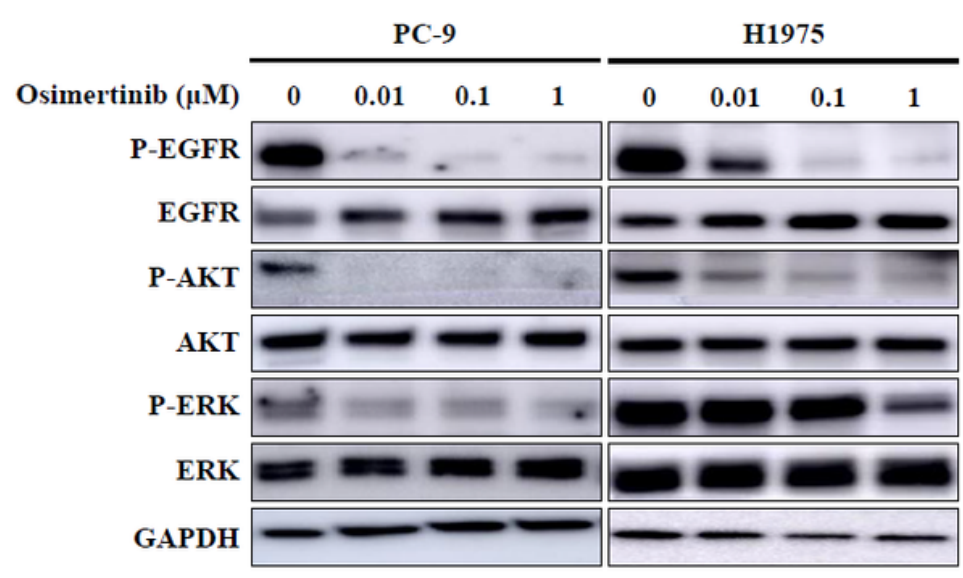

C

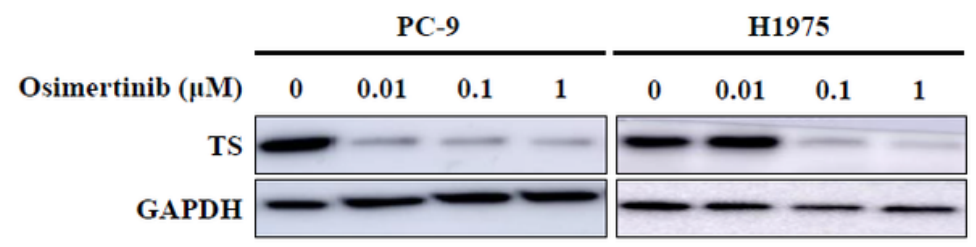

D

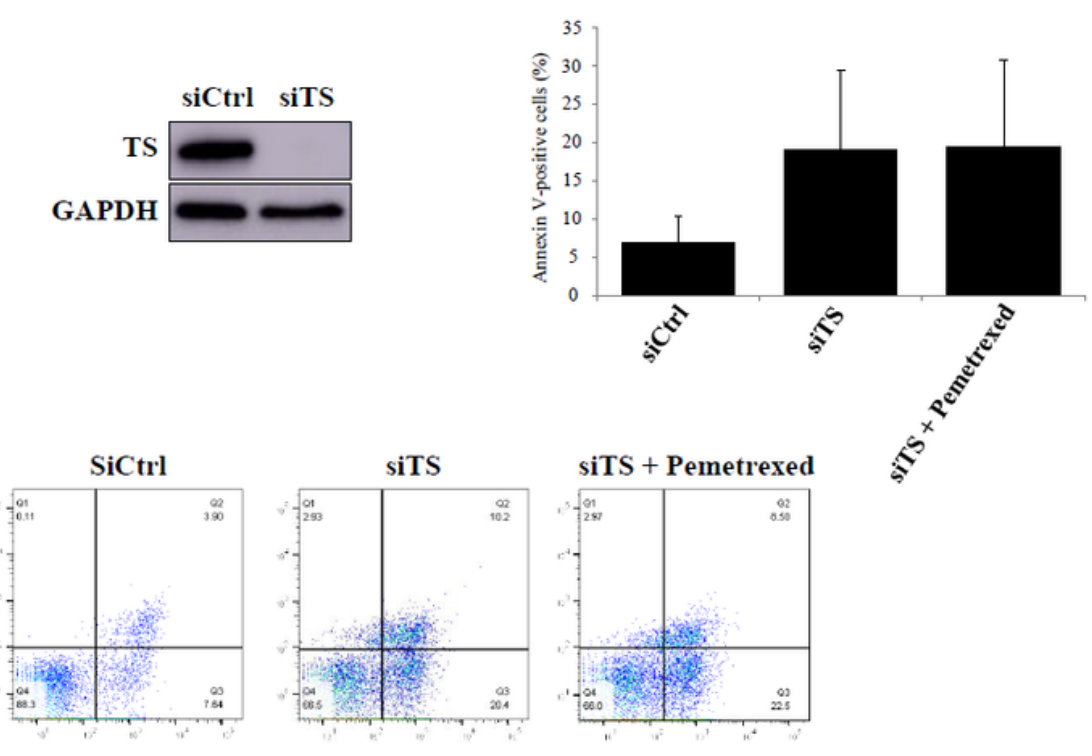

B
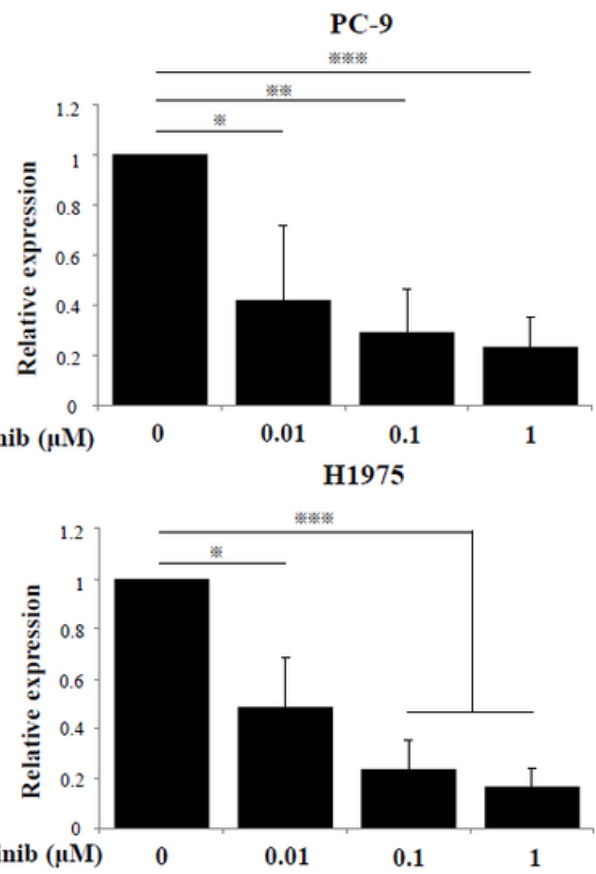

Figure 3 
Effects of thymidylate synthase (TS) on the antitumor effects of osimertinib in combination with pemetrexed. A, Protein levels of p-EGFR, EGFR, p-AKT, AKT, p-ERK and ERK were analyzed by Western blotting. B, TS expression by PC-9 and $\mathrm{H} 1975$ cells after exposure to osimertinib for $24 \mathrm{~h}$ was suppressed as determined by quantitative real-time reverse transcription PCR (qRT-PCR; $* p<0.05, ※ ※ p<0.01$, ※※ $\mathrm{p}<0.001)$. C, TS protein expression was analyzed by Western blotting. D, TS expression was significantly decreased after TS-specific siRNA treatment (upper). Percentages of apoptotic cells in PC-9 cells treated with TS-specific siRNA after treatment with pemetrexed at their half IC50 doses for 48 hours (lower). 


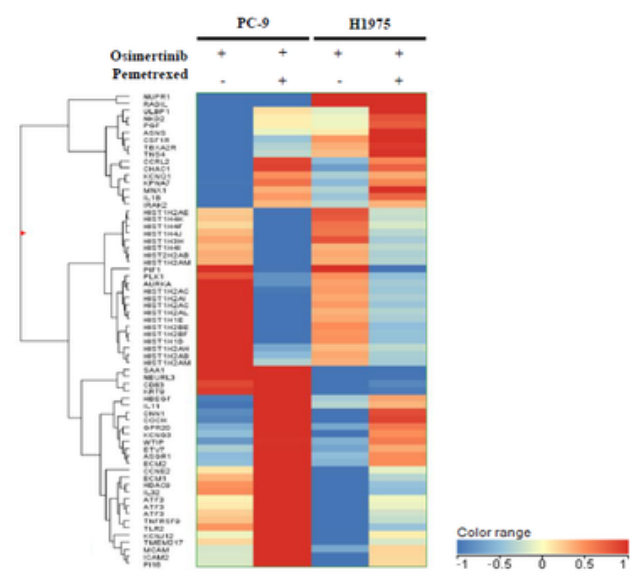

C

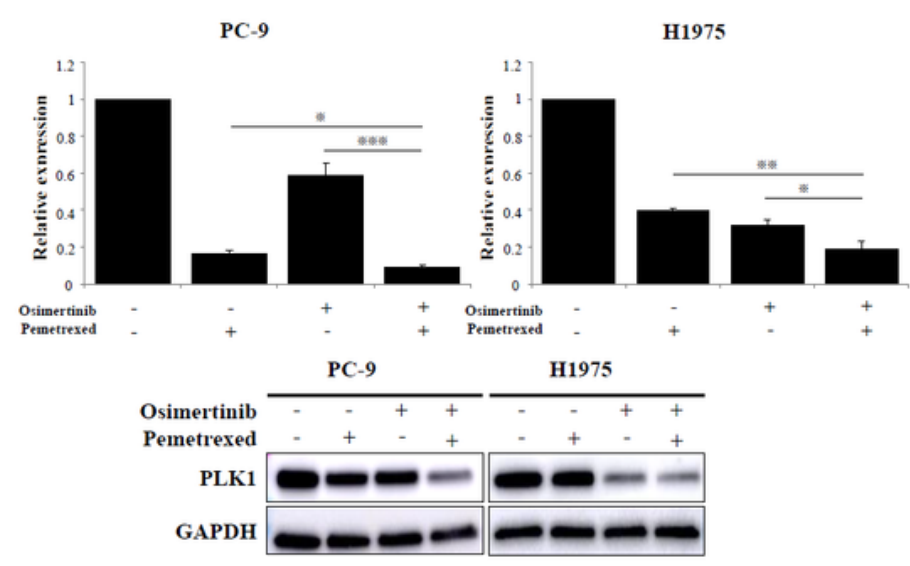

F, G

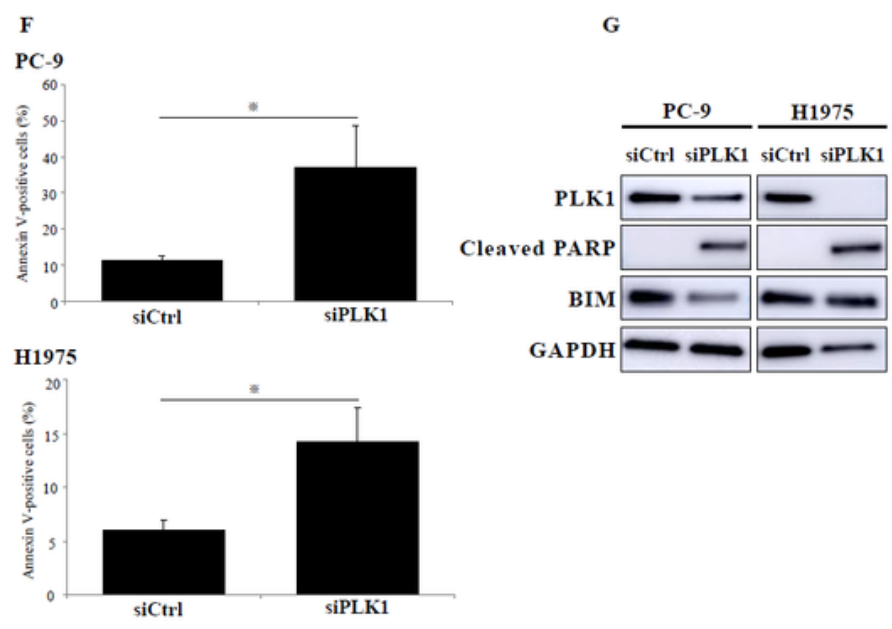

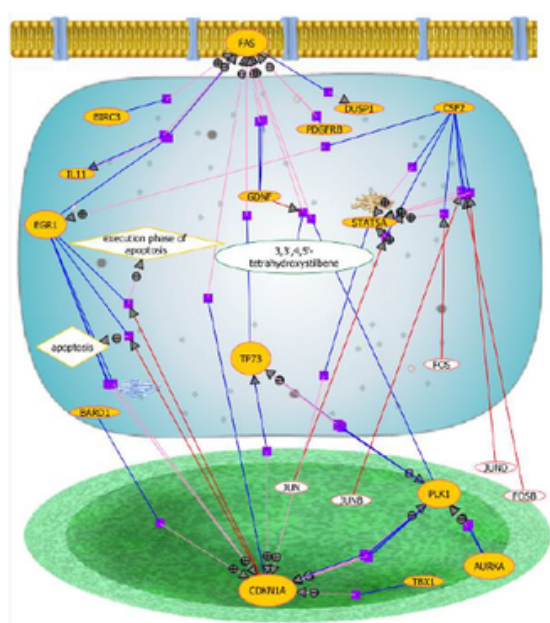

D

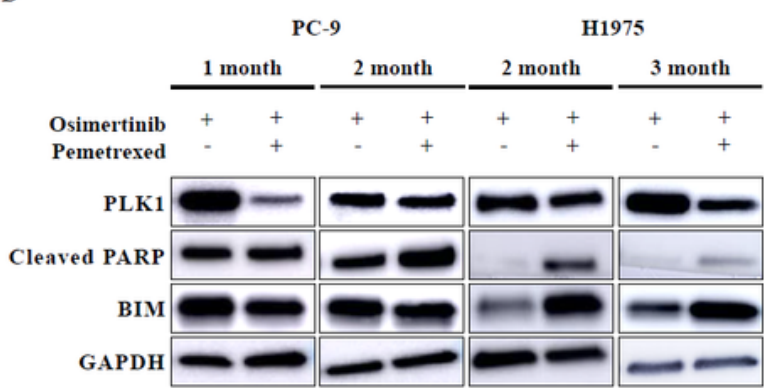

E

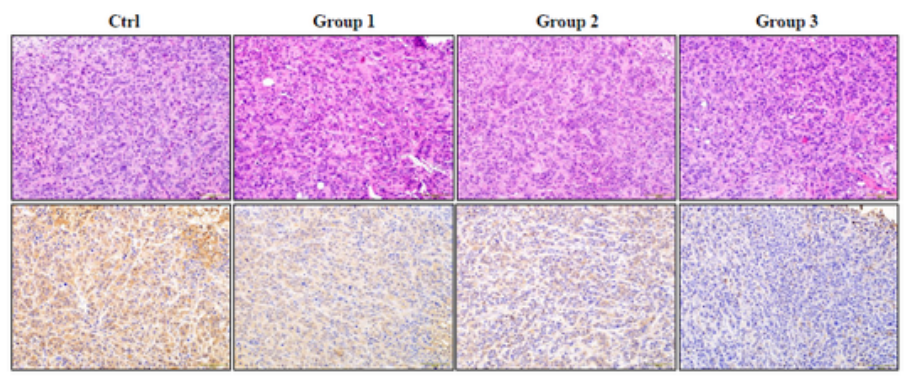

\section{Figure 4}

Downregulation of polo-like kinase 1 (plk1) is associated with antitumor effects of the combination of osimertinib and pemetrexed. A, Gene expression profiles of PC-9 and H1975 cells treated with osimertinib with or without pemetrexed. Heat map of genes whose expression fluctuated more than 3-fold. B, Pathway analysis for apoptosis regulation showing the importance of PLK1 and AURKA genes associated with apoptosis. C, Expression of plk1 in PC-9 and H1975 cells after exposure to osimertinib 
and pemetrexed for 24 hours. Suppression compared with osimertinib or pemetrexed alone as determined by quantitative real-time reverse transcription PCR (qRT-PCR) is depicted. $※ p<0.05, ※ ※ p<0.01, ※ ※ \cdots p$ $<0.001$. PLK1 protein was analyzed by Western blotting. D, PLK1 expression in cells treated by both osimertinib and pemetrexed before the emergence of resistance, relative to monotherapy. E, PLK1 expression in the PC-9 xenografted mouse tumor was analyzed by IHC. HE staining and IHC staining of PLK1 is shown. Staining xenografted mouse tumor treated with osimertinib and pemetrexed for PLK1 (scale bar $=100 \mu \mathrm{m}$ ). F, G, Percentages of apoptotic cells in PC-9 and H1975 cells treated with plk1-specific siRNA (※ p 0.05). Levels of apoptosis markers in PC-9 and H1975 cells treated with plk1-specific siRNA.

A

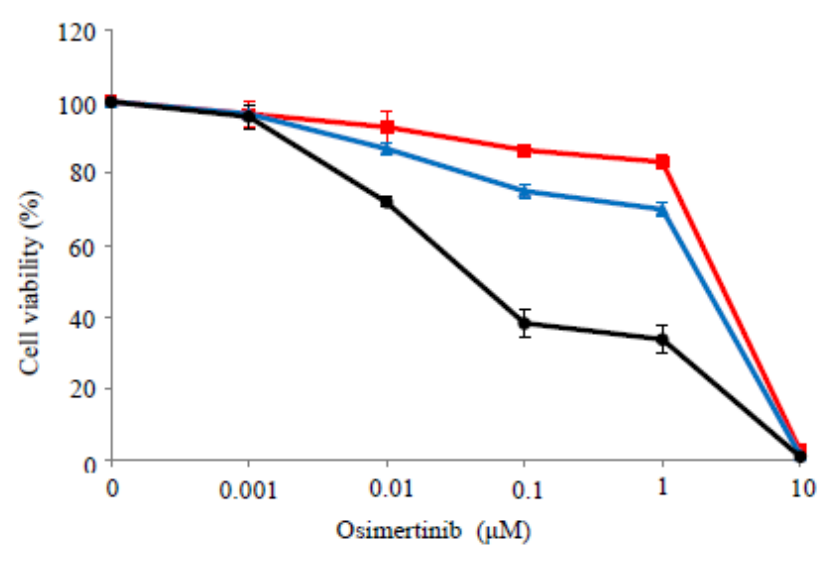

$\longrightarrow$ PC-9

$\longrightarrow$ PC-9OsiR

— PC-9OsiPEMR

C

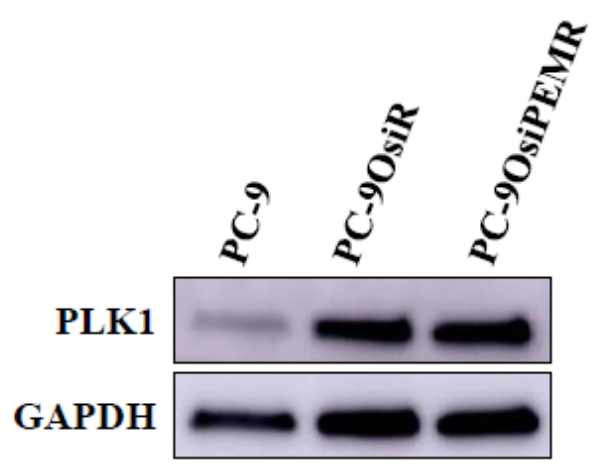

B

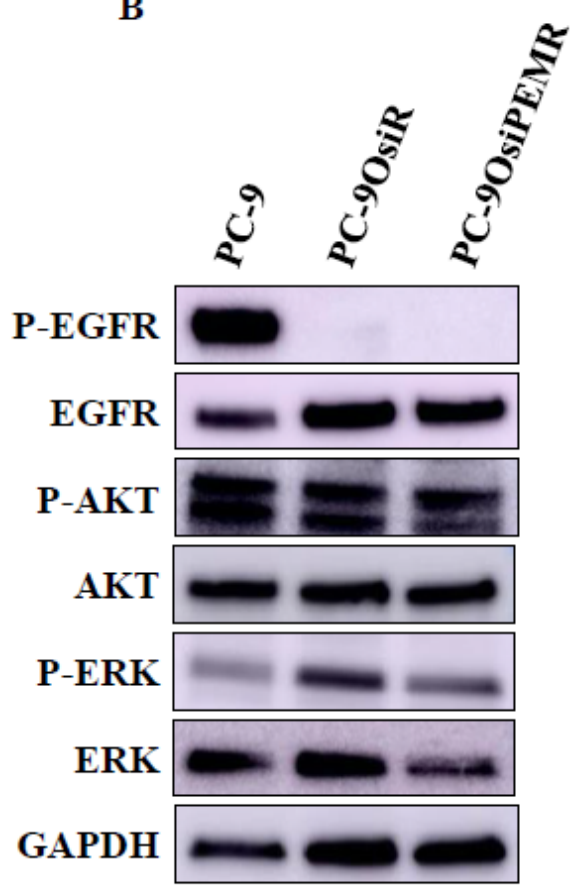

D

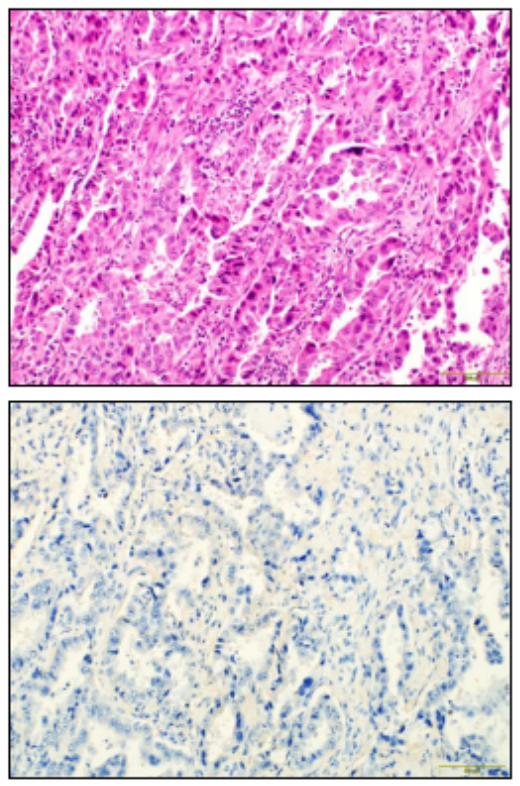

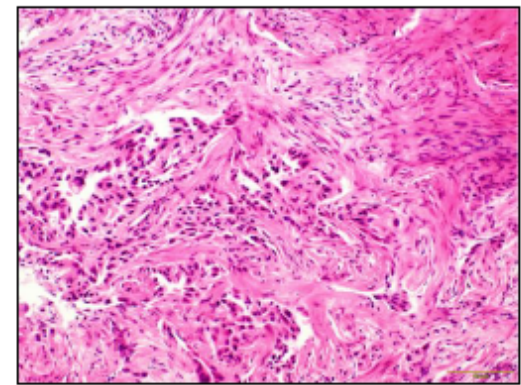

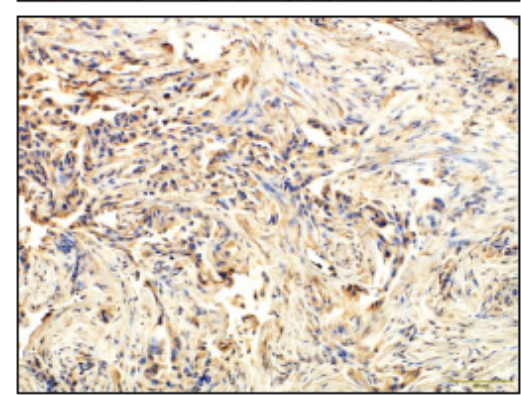

Figure 5 
PLK1 upregulation is implicated in acquired resistance to osimertinib and pemetrexed in EGFR-mutant cells and in a patient. A, MTT assays showing that PC-90siR and PC-90siPEMR are resistant to osimertinib. B, PC-90siR and PC-90siPEMR showing p-EGFR, p-AKT and p-ERK expression by Western blotting. C, PLK1 expression in PC-90siR and PC-90siPEMR. D, Hematoxylin and eosin staining in EGFRmutant lung cancer specimens from an NSCLC patient before (upper left) and after osimertinib+pemetrexed therapy (upper right). Staining for PLK1 IHC before treatment (lower left) and after osimertinib and pemetrexed therapy (lower right) (scale bar=100 $\mu \mathrm{m}$ ).

A

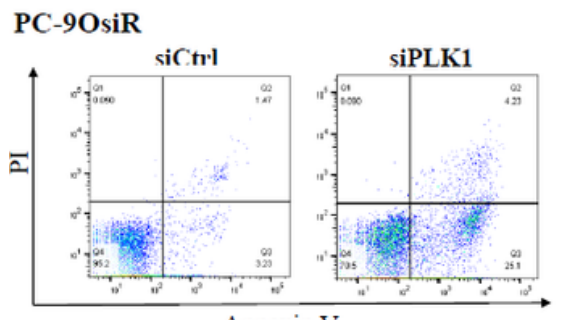

Annexin V

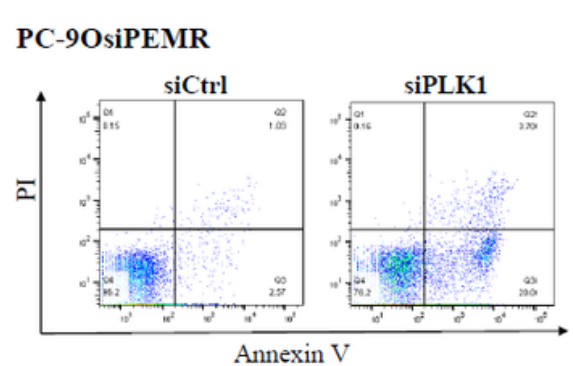

C

PC-9OsiR

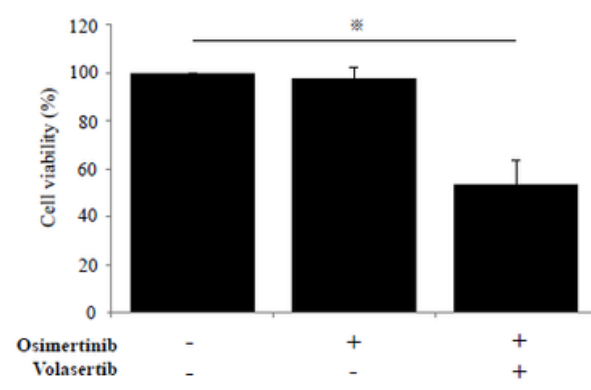

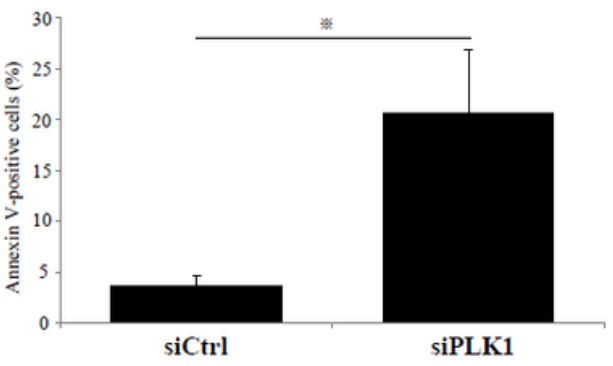

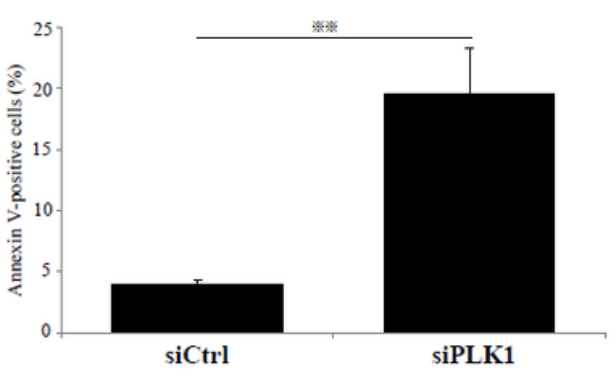

D
B

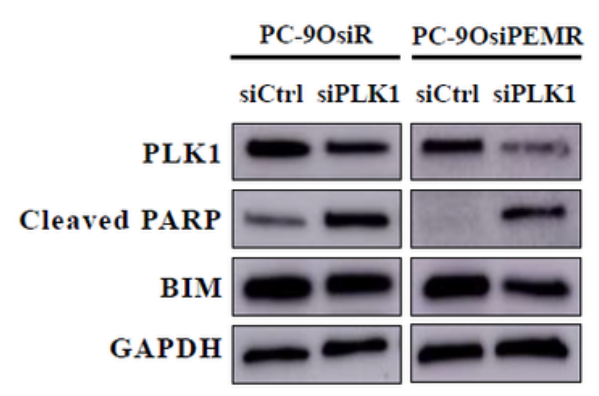

Figure 6

PC-9OsiPEMR

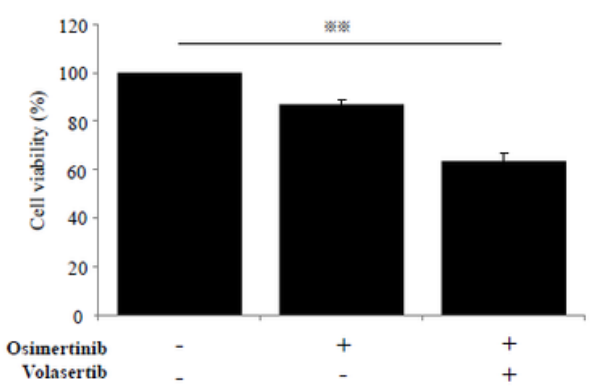

PC-9OsiR Ctrl Volasertib $\mathrm{Ctrl}$ Volasertib

Cleaved PARP

BIM GAPDH

Inhibition of PLK1 induced apoptosis in osimertinib-resistant cell lines and inhibition of proliferation. A, Percentage of apoptotic cells in PC-90siR and PC-90siPEMR cells treated with plk1-specific siRNA $(※ p<$ $0.05, ※ ※ p<0.01)$. B, Levels of apoptosis markers in PC-90siR and PC-90siPEMR cells treated with plk1specific siRNA. C, Cell viability of PC-90siR and PC-90siPEMR in response to volasertib (25 nM) compared to osimertinib $(100 \mathrm{nM})$ for 72 hours $(※ p<0.05, \approx ※ p<0.01)$. D, Volasertib-induced cleaved PARP expression in PC-90siR and PC-90siPEMR cells. 


\section{Supplementary Files}

This is a list of supplementary files associated with this preprint. Click to download.

- supplementaryinformation20210301.pdf 